
internationales

vol. 30 - n² | 2014

Composer (avec) la frontière. Passages, parcours

migratoires et échanges sociaux

\title{
Récits de traversée de la frontière entre l'Égypte et la bande de Gaza
}

Stories of Border Crossing between Egypt and the Gaza Strip

Historias de cruce de fronteras entre Egipto y Franja de Gaza

\section{Lorenzo Navone}

\section{OpenEdition}

Journals

\section{Édition électronique}

URL : https://journals.openedition.org/remi/6829

DOI : $10.4000 /$ remi.6829

ISSN : $1777-5418$

Éditeur

Université de Poitiers

\section{Édition imprimée}

Date de publication : 1 juin 2014

Pagination : 157-168

ISBN : 979-10-90426-22-1

ISSN : 0765-0752

\section{Référence électronique}

Lorenzo Navone, "Récits de traversée de la frontière entre l'Égypte et la bande de Gaza », Revue européenne des migrations internationales [En ligne], vol. 30 - n² | 2014, mis en ligne le 01 juin 2017 , consulté le 14 avril 2022. URL : http://journals.openedition.org/remi/6829 ; DOI : https://doi.org/ 10.4000/remi.6829 


\section{Note de recherche}

\section{Récits de traversée de la frontière entre l'Égypte et la bande de Gaza}

\section{Lorenzo Navone ${ }^{1}$}

L'objet de cette note de recherche est de décrire la fragmentation de l'espace social produit par la frontière "triangulaire " située dans la région du nord du Sinaï, au carrefour entre I'Égypte, Israël et la bande de Gaza. II s'agit ainsi de contribuer à la compréhension du mode de fonctionnement de cette frontière, à travers des témoignages de personnes qui l'ont franchie ou ont échoué dans l'entreprise ${ }^{2}$.

Notre expérience sur le terrain, l'observation des pratiques ainsi que les entretiens réalisés témoignent de la complexité de cette frontière, que l'on ne peut réduire à une métaphore qui l'interprète comme une membrane comprimée entre deux forces opposées : la tension entre ouverture et fermeture, intérieur et extérieur, inclusion et exclusion, crossing borders et reinforcing borders (Vila, 2000). Le compte rendu ${ }^{3}$ que nous proposons de nos enquêtes de terrain (août 2009, février-mai 2010, septembre 2011) se base sur des données ethnographiques recueillies dans la région frontalière et à proximité du point de passage de Rafah (Rafah Border Crossing), du côté égyptien. II décrit des évènements ainsi que des expériences que l'on peut considérer comme paradigmatiques liés aux regroupements ou aux séparations de familles fragmentées en raison de la frontière et tente de répondre aux questions suivantes : qui peut franchir cette frontière ? Qui, en revanche, n'est pas autorisé à le faire ? Quand la frontière est-elle ouverte et pourquoi ? Quels "régimes de perméabilité " montre-t-elle et quelles hiérarchies de passage génère-t-elle et enfin quels en sont les effets ?

Les récits et les expériences de traversée (ou de non-traversée) de la frontière constituent le fil directeur de la narration.

\footnotetext{
1 PhD, Università Degli Studi di Genova, Disfor, Corso A. Podestà 2, Terzo piano, stanza 3A4, 16128, Genova, Italie ; lo.navone@gmail.com

2 Ce texte a été rédigé avant l'opération "Bordure protectrice " qui s'est déroulée durant l'été 2014.

3 L'observation et la participation, par immersion, aux événements significatifs de la vie dans cet espace liminal, les douze entretiens réalisés et de nombreuses discussions informelles avec les personnes rencontrées du côté égyptien de la frontière (organisation du voyage, déplacement vers le lieu de passage, points de contrôle égyptien, attente de l'ouverture du terminal d'un côté ou de l'autre de la frontière, nuits passées dans la rue, etc.) constituent la matière de ce compte rendu.
} 


\section{Préliminaires}

La frontière israélo-égyptienne possède une histoire particulière. Cette ligne, déjà frontière inter-ottomane, tracée dans le désert entre la mer Méditerranée et la mer Rouge, de Rafah à Taba, devient une frontière politique en 1906, suite à l'accord signé entre l'Empire Ottoman et l'Égypte qui mit fin au conflit concernant le Sinaï. Limite sud-occidentale de la Palestine sous mandat britannique (1920-1948), sa position actuelle est le résultat de trente années de conflits (1948-1979) où la frontière a correspondu à plusieurs reprises à la ligne de front, prenant donc la forme d'une véritable frontière mobile. Un bref rappel de ses mouvements peut se révéler utile pour comprendre son impact sur le présent. En 1956, la crise de Suez offre l'opportunité à Israël d'envahir et d'occuper militairement, avec le soutien de la France et du Royaume-Uni, la péninsule du Sinaï avant le retrait définitif d'Israël et de la relève par la Force d'urgence des Nations Unies (FUNU). À cette date, la frontière entre I'Égypte et Israël se trouvait près du canal de Suez. En 1967, durant la guerre des Six-Jours (du 5 au 10 juin), Israël étend à nouveau ses propres frontières méridionales jusqu'à occuper et coloniser totalement le Sinaï (outre la bande de Gaza, la Cisjordanie et le plateau du Golan). La frontière de l'Égypte correspond au canal de Suez, ligne de cessez-le-feu. Le problème du retrait des Territoires occupés en 1967 entraînera en 1973 un nouveau conflit, la guerre du Kippour (du 6 au 24 octobre), au terme de laquelle l'Égypte reprend le contrôle du canal de Suez, alors que le Sinaï reste occupé par Israël. Les accords de Camp David en 1978 et le traité de paix israélo-égyptien de 1979 marquent le retour progressif de la frontière à sa position de départ, une position qui, dans ses grandes lignes, reprend le tracé défini trente années auparavant suite à l'armistice de Rhodes, qui mit fin à la première guerre arabo-israélienne (1948-1949).

De 1948 à 1967, la bande de Gaza se trouve donc sous administration égyptienne, tandis que de 1967 à 1979 le Sinaï est occupé par l'armée israélienne. Ces deux circonstances ont concouru à réduire la discontinuité politique entre la bande de Gaza et le Sinaï dont l'interface a été plus ou moins perméable jusqu'en 1982, année du retrait israélien de la péninsule, de la restitution totale du Sinaï à l'Égypte et de la division, marquée par la construction d'un mur de séparation, de la ville de Rafah en deux secteurs, I'un égyptien et l'autre palestinien (Hanafi et Sammartin, 1996).

De 1982 à 2005, le passage de Rafah a été gouverné par Israël, qui continuait à occuper militairement et coloniser la bande de Gaza. Actuellement, le passage de Rafah est pratiquement fermé depuis plusieurs années : suite au désengagement israélien de la bande de Gaza (2005), l'Autorité nationale palestinienne (ANP) a pris progressivement le contrôle de la partie du passage relevant de sa compétence contrôlée précédemment par Israël, mais après la victoire électorale du Hamas (en janvier 2006), l'échec des négociations pour un gouvernement d'unité nationale et le début, en 2007, d'une guerre civile entre les mouvements politiques Fatah et Hamas, la mission civile des observateurs de I'Union européenne (EU BAM Rafah, Mission de I'Union européenne d'assistance à la frontière entre Gaza et l'Égypte au point de passage de Rafah) s'est retirée du terminal, Israël a imposé un état de siège à la bande de Gaza et l'Égypte a matériellement " fermé la porte ". 
La dizaine de kilomètres qui sépare l'Égypte de la bande de Gaza est un espace encore peu étudié sous l'angle ethnographique (Hanafi et Sammartin, 1996), bien qu'il constitue, du point de vue historique et géographique, une partie de la frontière internationale entre l'Égypte et l'État juif. Situé à l'extrémité nord de la ligne frontalière, le passage de Rafah représente aujourd'hui la seule " frontière extérieure " des Territoires occupés où il n'y a pas de présence israélienne ${ }^{4}$. Aujourd'hui, le passage de Rafah est ouvert au transit des personnes de manière sporadique et aléatoire, environ trois ou quatre jours par mois, et en dehors du cadre juridique et technique établi au cours de la période du processus d'Oslo ${ }^{5}$. Du côté égyptien l'ouverture du passage n'est annoncée que quelques jours auparavant par les autorités, une semaine maximum, à travers les médias locaux; les dates exactes et la durée de l'ouverture restent cependant imprévisibles.

\section{Photo 1 : Rafah, Égypte, la queue devant le terminal, août 2009}

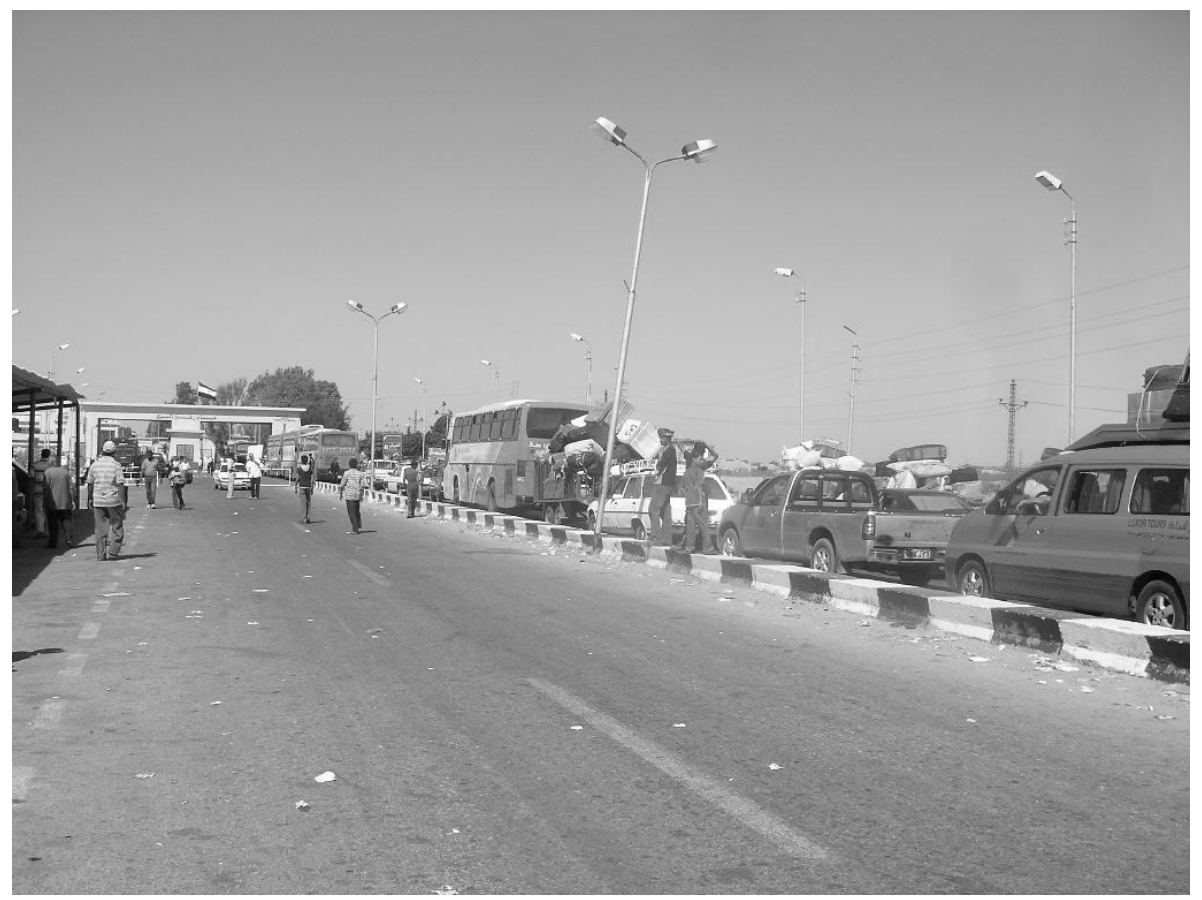

Crédit : Lorenzo Navone.

4 Le pont Allenby (ou Allenby Bridge Border Crossing) se trouve entre la Jordanie et la zone C (sous contrôle israélien) de la Cisjordanie, près de Jéricho. Ce terminal est administré par l'Autorité aéroportuaire israélienne, contrairement au terminal de Rafah, qu'Israël ne dirige plus depuis 2005 (théoriquement et physiquement). Nous pouvons donc considérer Rafah comme étant la seule frontière extérieure des Territoires occupés.

5 Les accords d'Oslo (protocole des passages) et ceux qui ont suivi avaient établi un cadre et des procédures de passage qui n'ont plus été respectés après la prise du pouvoir de la bande de Gaza par le Hamas (2007). 
La seule condition nécessaire pour traverser le passage est la possession d'un passeport palestinien en cours de validité (sauf pour le passage des " VIP ${ }^{6}$ "). Pendant les jours d'ouverture, nombreux sont celles et ceux qui se positionnent le long de la route principale qui mène au terminal, formant une longue file de personnes, de véhicules et de bagages en tout genre. C'est dans ce contexte que s'est déroulé notre travail sur le terrain.

Le dispositif frontalier filtre, sélectionne et gouverne les personnes selon des dynamiques de refoulement, de ralentissement, d'attente et d'expulsion qui correspondent à des situations vécues le plus souvent par nos interlocuteurs.

\section{Le passage interdit}

Maysa est une jeune Américaine d'origine palestinienne que nous avons rencontrée au terminal de Rafah alors qu'elle attendait, en compagnie de sa mère âgée, de passer la frontière afin de rendre visite à ses parents à Gaza. Maysa, contrairement à sa mère, ne possédait que son passeport américain, certainement plus utile qu'un passeport palestinien pour passer toutes les frontières du monde à l'exception du passage de Rafah, où la possession d'un document de voyage palestinien est essentielle pour la traversée. Maysa, refoulée à l'entrée, n'avait pas d'autre choix que de retourner au Caire puis de là, rentrer au Texas, ou bien d'attendre la prochaine ouverture du point de passage pour tenter à nouveau sa chance : dans les deux cas, elle devait renoncer à l'idée de passer le Ramadan avec les parents de sa mère, à Gaza.

"- Est-ce que quelqu'un t'a dit quand est-ce que la frontière sera de nouveau ouverte?

- Non, ils n'ont rien dit. D'après ce que j'ai entendu, ils l'ouvriront de nouveau dans un mois, un mois et demi.

- Mais tu as déjà un billet de retour?

- Non, nous n'avons pas encore réservé, parce que nous avions peur que la sortie soit retardée et donc de perdre le billet. Nous avons donc laissé le retour ouvert. Je veux que ma mère voit ses parents, passe du temps avec eux et puis, dès que la frontière rouvre, je veux juste m'en aller.

- Tu es déterminée à entrer à Gaza?

- Oui, je veux connaître la famille de ma mère, je ne l'ai jamais vue. C'est une partie de ma vie que je ne connais pas, tu comprends? Je ne les ai jamais connus, ses frères et ses sœurs. Je veux connaitre cet aspect de ma vie, tu comprends? Je suis curieuse, donc je vais rester ici cette nuit, et demain je vais essayer d'entrer. J'ai peur. Je ne me suis jamais trouvée dans une situation de ce genre. Mais je ne veux pas me rendre".

6 Selon l'Accord réglant les déplacements et le passage (Agreement on Movement and Access) et les principes convenus concernant le passage de Rafah (Agreed Principles for Rafah Crossing), du 15 novembre 2005, signé entre Israël et l'Autorité palestinienne, certaines catégories de personnes, désignées sous le nom de VIP ou " cas exceptionnels " se rapportant aux membres du corps diplomatique, aux investisseurs internationaux, aux membres d'organisations internationales et non-gouvernementales et aux cas humanitaires, peuvent traverser le passage de Rafah, à condition de présenter une notification préalable faite au moins quarante-huit heures avant. Cette notification est formalisée par écrit par la partie palestinienne à la partie israélienne. 
L'histoire de Maysa représente un des nombreux cas de refoulement à la frontière, ou de non-accès, dont nous avons été témoin au terminal de Rafah. Comme Maysa, en effet, plusieurs Palestiniens de différentes nationalités se sont vu refuser l'entrée et ont été refoulés au terminal, en raison de l'absence d'information circulant sur le fonctionnement de la frontière et sur les règles de franchissement. Nous avons aussi rencontré plusieurs Palestiniens avec des passeports américains ou de différents pays européens qui, une fois à la frontière, ont réalisé ne pas être en possession des documents nécessaires pour la traverser ; ils n'avaient que le passeport du pays où ils étaient résidents. La plupart du temps, ils passaient quelques jours dans la zone frontalière, parfois en campant quelques nuits sur le bord de la route, avec l'espoir de traverser tôt ou tard la frontière peut-être même le dernier jour de l'ouverture du passage, avant de repartir au Caire pour tenter d'obtenir un permis spécial auprès de I'Ambassade palestinienne.

\section{L'attente prolongée}

Walid est un homme d'environ trente ans, originaire de Khan Younis, dans la bande de Gaza. Diplômé, il a travaillé dans l'administration publique à Gaza pour l'Autorité palestinienne, avant l'arrivée au pouvoir du Hamas. Suite à cet événement, l'embargo international et la dégradation des conditions de vie dans l'enclave palestinienne ont convaincu Walid d'abandonner la bande de Gaza pour l'Égypte, où se trouvait une partie de sa famille. En possession d'un visa valable un an, il a déménagé au Caire où il n'a cependant pas pu retrouver un emploi. Sa démarche n'ayant pas abouti il est donc conduit, contre sa volonté, à prendre la décision de retourner à Gaza, pour ne plus peser économiquement sur sa famille. Quand nous l'avons rencontré au passage de Rafah, il faisait partie des personnes qui, bien que possédant un passeport palestinien, n'ont pas réussi à franchir la frontière parce qu'elles n'étaient pas à la bonne place dans la file d'attente qui petit à petit s'était formée, mais aussi parce qu'elles ne possédaient ni les connaissances ni les moyens économiques qui leur auraient éventuellement permis d'éviter l'attente ${ }^{7}$.

Après quelques jours d'attente entre El-Arich et Rafah, et plusieurs renvois, Walid a décidé de se rendre à El-Arich, la capitale du gouvernorat du Sinaï Nord, et d'attendre l'ouverture prochaine du point de passage. Cependant, cette attente forcée implique des coûts supplémentaires qui n'étaient pas prévus dans le budget de la famille de l'autre côté de la frontière.

\footnotetext{
7 L'espace-frontière et les milliers de personnes qui attendent sont, pour ceux qui se présentent comme détenteurs du savoir pratique lié au passage de la frontière, c'està-dire les Bédouins du Sinaï, une grande opportunité pour gagner de l'argent. Ces derniers peuvent fournir et vendre aux candidats qui veulent traverser la frontière tout type de fourniture utile pour la maison et un certain nombre de matériaux de construction (comme l'acier, le ciment, etc. qui sont plutôt difficiles à trouver à Gaza) à des prix plus élevés qu'en Égypte continentale. Ils se présentent souvent à la frontière comme routiers, chauffeurs de taxi, médiateurs, porteurs, etc. Ils peuvent échanger de manière informelle les devises qui circulent dans la région (shekel, dollars, euros, dinars). Mais surtout, à travers un réseau compliqué d'intermédiations, d'alliances frontalières et une "gestion négociée des règles du désordre " (Palidda, 2002) avec les autorités locales, les Bédouins qui contrôlent le système des tunnels transfrontaliers - dont I'apparition constitue une constante dans les conflits et un héritage typique des après-guerres (Andreas, 2004) - offrent la possibilité de contourner la traversée de la frontière aux marchandises et aux personnes en échange de sommes importantes.
} 
Des étudiants palestiniens ${ }^{8}$ " en déplacement " vivent plus ou moins le même type de situation. Ainsi ceux qui fréquentent I'université du Sinaï, une université privée de El-Arich ${ }^{9}$ et qui souhaitent rendre visite à leur famille se trouvent bien souvent empêchés dans leur entreprise alors que les horaires d'ouverture de la frontière sont aléatoires. L'absence de règles claires sur les priorités et la direction de la traversée et donc l'impossibilité matérielle de planifier l'aller ou le retour, les amènent à attendre la pause estivale pour se rendre à Gaza afin de ne pas risquer de rater des cours ou des sessions d'examens. Le temps d'attente peut se prolonger sur plusieurs mois et chaque visite ne peut pas durer moins d'un mois, l'intervalle de temps minimum qui sépare deux ouvertures.

\section{L'attente à la sortie}

Certaines personnes que nous avons rencontrées au point de passage de Rafah n'attendaient pas pour entrer à Gaza, soit parce qu'elles n'étaient pas en possession des papiers nécessaires soit qu'elles n'avaient pas l'intention de traverser. Ces personnes, en effet, comme Wael, se trouvaient à la sortie en attente de leurs proches.

Wael est né à Gaza et a vécu plusieurs années au Koweït. Après la guerre du Golfe en 1990, il a abandonné le pays pour s'installer aux États-Unis, où ses enfants sont nés et où il exerce le métier de charpentier. Dans l'intention de retourner avec toute sa famille dans un pays arabe, il souhaitait que ses jeunes enfants soient inscrits au Bureau de l'état civil palestinien à Gaza. Pour ce faire, il fallait qu'ils se rendent avec leur mère dans la bande de Gaza pour procéder aux démarches administratives ${ }^{10}$. Pendant les trois ou quatre jours d'ouverture du point de passage de Rafah, en août 2009, nous avons rencontré Wael alors qu'il attendait la sortie de sa famille de l'enclave palestinienne et qu'il résidait dans le Sinaï depuis plus d'un mois ; la famille de Wael n'avait pas réussi à franchir la frontière à la première tentative et il avait dû louer un appartement à El-Arich en attendant la prochaine ouverture du passage ${ }^{11}$.

8 Plusieurs jeunes de Gaza, appartenant à la classe moyenne, achèvent leurs études universitaires à l'étranger, où les structures de recherche sont meilleures que dans la bande de Gaza, et où se déplacer à l'université n'est pas un parcours semé d'embûches comme dans les Territoires occupés, où l'absence intermittente d'énergie (électricité, diesel et essence à usage public et privé), les opérations militaires israéliennes à grande échelle, la présence des drones et les conflits armés entre factions politiques palestiniennes opposées sont plutôt fréquents et ne favorisent pas la recherche ni l'accès aux études et aux structures académiques.

9 Selon l'université, environ $17 \%$ des inscrits sont palestiniens (http://www.su.edu.eg). El-Arich se trouve à environ une cinquantaine de kilomètres de Gaza.

10 Le fonctionnement du registre de la population palestinienne est contrôlé par le "Registry and Passport Department " du ministère de I'Intérieur israélien, qui exige l'inscription des enfants dès les premières années de leur vie. "Le ministère de l'Intérieur israélien conserve la gestion du registre des populations et le monopole de l'émission des cartes d'identité palestiniennes. Il peut ainsi suivre et affecter les trajectoires spatiales (droit de résidence dans une enclave, permis de déplacement) et sociales des Palestiniens (mariage), ainsi que de multiples aspects de leur vie quotidienne (obtention d'un permis de conduire, immatriculation de véhicule, etc.) " (Parizot, 2009 : 63-64).

11 La sortie dépend de l'ouverture intermittente du passage de la frontière et de la priorité d'embarquement que les candidats acquièrent dans les bus qui mènent de Gaza City au terminal de Rafah. Les listes de sortie sont contrôlées par le Hamas et il n'y a pas de véritable certitude sur la date exacte de la traversée du point de passage, avec les conséquences économiques que cela peut comporter. 
La dernière nuit avant la fermeture du passage, Wael arrive enfin à retrouver sa famille, mettant ainsi fin à une attente de plus d'un mois.

L'histoire d'Hassan est encore plus compliquée : Palestinien originaire de la Cisjordanie et citoyen américain, Hassan était en vacances en Égypte avec ses fils, sa belle-sœur et sa femme, tous originaires de Gaza. Entrées dans la bande de Gaza pour enregistrer leurs enfants au Bureau de l'état civil et rendre visite à leur famille, les deux femmes étaient convaincues de pouvoir sortir après quelques jours. Cependant, les propos d'Hassan, à la sortie des deux femmes après plus d'un mois d'attente, témoignent d'une véritable odyssée :

" J'ai décidé pendant nos vacances au Caire, quand j'ai appris que la frontière était ouverte, de les accompagner pour qu'elles aillent à Gaza. Et ceci fut la plus grande erreur que j'aie jamais commise. [...] Maintenant nous sommes assis ici, tous ensemble, un mois et demi après leur entrée. J'ai passé trois jours à attendre, ici, à la frontière. Chaque jour, elles m'appelaient à partir de 5 h 30 du matin en me disant qu'elles étaient dans le bus, mais ils ne les faisaient pas descendre avant $19 \mathrm{~h}$. Il y a ma femme et mes trois enfants âgés de deux, quatre et six ans ainsi que sa sœur, enceinte de quatre mois, et sa petite fille de deux ans. Après avoir tenté de sortir de cette façon pendant trois jours, elles ont finalement réussi à sortir ce soir : le dernier jour d'ouverture de la frontière, à minuit. Je suis heureux qu'elles soient arrivées à sortir et j'espère que quelque chose va changer,

j'espère que raconter mon histoire sera utile à d'autres personnes ".

Des récits comme ceux de Walid, Wael et Hassan ne sont pas rares parmi les personnes présentes dans la file d'attente au point de passage. Ainsi des ressortissants égyptiens comme Oum Thaer peuvent vivre les mêmes conditions d'attente de l'autre côté de la frontière. Âgée d'une quarantaine d'années, elle est native du delta du Nil et vit à El-Arich avec ses deux enfants, dans un immeuble de trois étages dont elle est propriétaire. Après s'être mariée en Palestine avec un Gazaoui qu'elle a connu en Égypte, elle a passé plusieurs années dans la ville de Gaza avec son mari et ses enfants, tous nés dans la bande de Gaza. Ce mariage mixte ne naît pas d'une histoire migratoire particulière. II s'agit au contraire d'une situation courante en Égypte et ce type d'union ne rencontre pas d'obstacles de nature politique, linguistique ou même religieuse ; l'Égypte et la bande de Gaza sont deux pays qui entretiennent depuis toujours des relations de réciprocité. Cependant, le contexte historique particulier fait que cette " famille transnationale " doit sans cesse affronter des problèmes bureaucratiques en raison de l'occupation israélienne, dès le début de la première Intifada (1987-1993) :

" - J'étais dans une situation paradoxale car, sans documents officiels, mes enfants étaient considérés aux yeux de loi israélienne Égyptiens dans la bande de Gaza et Palestiniens en Égypte. Je ne pouvais pas rester à Gaza plus de six mois, je devais sortir et rentrer à chaque fois. Le terminal était sous le contrôle des Israéliens : selon eux, les enfants étaient les miens, parce que j'étais égyptienne, donc ils avaient ma nationalité, mais leur naissance a été enregistrée dans la bande de Gaza, où je ne pouvais pas travailler. Après le troisième enfant, j'ai décidé de rendre visite à ma famille en Égypte, mais à mon retour, les Israéliens ne m'ont pas laissé passer. J'avais un document de voyage, mais pas de passeport, ce qui me permettait seulement d'entrer à Gaza, donc en Israël à l'époque. - Et ensuite? 
- J'étais dans une situation terrible : les enfants ne pouvaient pas aller à l'école en Égypte parce qu'ils étaient considérés Palestiniens ${ }^{12}$, mais ils ne pouvaient pas non plus entrer en Palestine. J'étais dans un endroit, mon mari dans un autre. D'autres personnes dans

la même situation que moi ont dû divorcer car ils n'ont pas réussi à trouver d'autres solutions pour régler le problème. Mon mari est avocat et a des relations, il a fait tout son possible pour me faire rentrer à Gaza avec nos trois enfants. Après, je suis restée

pendant quatre ans dans la bande de Gaza parce que mon mari ne voulait pas que je sorte, craignant que je ne puisse plus rentrer. Pendant toute cette période, j'ai fait plusieurs fois la demande pour obtenir la nationalité, mais cela s'est avéré difficile. J'ai réussi à obtenir une carte d'identité palestinienne lorsque, après 1993, Israël a finalement autorisé l'Autorité palestinienne à délivrer des documents à quelques personnes. J'ai reçu un "document vert"13, ce qui m'a donné la possibilité de me déplacer. J'ai eu de la chance parce que je n'ai pas bougé de Gaza durant quatre ans. D'autres en revanche, qui ont été mariés pendant plus longtemps, n'ont pas obtenu la nationalité parce qu'ils faisaient sans cesse des allers-retours. J'ai eu beaucoup de chance car avec le document vert c'était facile de traverser la frontière, qui était ouverte vingt-quatre heures

sur vingt-quatre ".

Lorsque nous avons fait sa connaissance, Oum Thaer vivait à El-Arich depuis plusieurs années : après l'arrivée au pouvoir du Hamas, elle et son mari avaient choisi El-Arich comme lieu de résidence en raison de sa situation de ville frontière entre l'Égypte et la bande de Gaza. Elle a vécu là avec ses deux plus jeunes enfants, en attendant l'arrivée de son mari et des autres enfants qui se trouvaient dans la bande de Gaza en attente d'une permission de traverser la frontière afin de s'établir de façon plus ou moins permanente en Égypte :

" - Nous sommes venus ici et nous avons essayé de demander le droit de résidence pour tous les enfants parce que je suis égyptienne. Pour eux, j'avais déjà essayé de demander un permis de séjour, et nous l'avions obtenu, mais pour le conserver nous aurions dû venir ici au moins une fois par an, donc nous l'avions perdu parce que nous étions restés à Gaza. J'ai essayé de renouveler le permis de séjour, mais les autorités égyptiennes ont refusé parce qu'ils ont dit qu'ils étaient entrés illégalement. Donc, ils étaient sans-papiers et ils ont tous décidé de retourner [à Gaza]. Moi, je suis restée ici avec les deux plus petits. - Donc, tu es restée seule?

- Mon mari a essayé de sortir de Gaza afin de subir une opération des yeux : il a fallu six mois pour obtenir l'autorisation, mais pour les enfants il n'y a rien eu à faire.

- Pourquoi es-tu restée ici?

- J'ai décidé de vivre ici parce que c'est près de la maison. Mon mari devrait arriver ici demain, pour essayer entre autres de faire quelque chose avec l'Ambassade palesti-

12 La loi égyptienne sur la nationalité est un ensemble complexe de Jus sanguinis et de Jus soli. Ce n'est que depuis 2004 qu'un amendement de cette loi a désormais rendu effective l'acquisition de la nationalité pour les personnes nées à l'étranger de mère égyptienne (http://en.wikipedia.org/wiki/Egyptian_nationality_law).

13 Les détenteurs de la Green ID Card sont également titulaires d'un document de voyage et peuvent se déplacer à l'intérieur de la bande de Gaza ou en Cisjordanie, mais pas de I'un à I'autre, parce qu'ils ne peuvent pas traverser l'État d'Israël. La population arabe résidant dans la Palestine historique a quatre types de ID Card: Blue Card, avec le passeport israélien et la nationalité (les Arabes israéliens) ; Blue Card sans passeport israélien (les habitants de Jérusalem-Est) ; Green Card (les habitants des Territoires occupés) ; Orange Card (les habitants des Territoires occupés avec des autorisations spéciales). Plusieurs milliers de Palestiniens ne sont munis d'aucun type de ID Card. 
nienne afin de faire sortir tous les enfants. Mais même si on réussissait à les faire sortir, ce ne serait pas la fin de nos souffrances, car ils ne sont pas reconnus comme des Égyptiens et ils doivent payer plus pour étudier. Un Égyptien doit payer environ 160 livres égyptiennes de droits de scolarité, un étranger environ 18000 , pour l'université publique !

Mon troisième fils a perdu une année d'étude pour tenter de s'inscrire à l'université en Égypte.

- Et les petits?

- Seulement la petite a le droit à la nationalité égyptienne parce qu'elle est née après 2005, lorsque la loi sur la nationalité et la migration a changé. Si Gaza est aussi désespérée comme on le voit à la télévision, pourquoi les Égyptiens ne traitent pas les enfants des Égyptiens comme des Égyptiens?".

Oum Thaer attendait donc chaque jour à El-Arich le moment où la famille serait enfin réunie ; entre temps, elle devait élever et entretenir toute seule deux enfants en bas âge.

Ce que met en évidence la situation d'Oum Thaer et de sa famille c'est la facilité avec laquelle, une fois la frontière franchie, il est possible de rester coincé d'un côté ou de l'autre, dans l'attente d'un regroupement familial dont on ne sait quand il aura lieu et s'il aura lieu.

La faible perméabilité de la frontière et ses règles, souvent perçues par les acteurs concernés comme n'étant que des entraves arbitraires à la circulation des personnes, ont un impact important sur la vie et le statut des personnes confrontées à ce dispositif. L'humiliation que ressentent les personnes qui se trouvent dans cette attente "définitivement temporaire " (Rahola, 2003) peut être lue comme une punition réservée aux transgresseurs de la frontière et des souverainetés que celle-ci sépare (Khosravi, 2010 : 67).

\section{La séparation forcée}

D'une certaine manière, I'histoire d'Oum Thaer introduit à la quatrième possibilité de traversée, de la frontière. Nous avons ainsi rencontré, dans nos enquêtes, un nombre considérable de personnes contraintes de se séparer de leur famille et de rompre leurs attaches affectives pour traverser la frontière, dans un sens comme dans l'autre.

Les exilés palestiniens du Fatah en Égypte ${ }^{14}$, par exemple, mais aussi quelques "Égyptiens " font partie de ces personnes. Suheir est une enfant en âge d'être scolarisée que nous avons rencontrée un matin avec sa mère et sa jeune sœur dans un taxi collectif qui allait de El-Arich jusqu'au terminal de Rafah. Pendant le voyage, la mère des deux filles, Iman, trente ans, nous a décrit leur situation : Suheir, comme sa sœur cadette, est née en Égypte, à Mansura, de parents palestiniens résidant régulièrement en Égypte, mais qui ne sont pas titulaires de la pleine nationalité ; dans ce cas, la loi égyptienne sur la nationalité ne permet pas aux enfants d'accéder de façon quasi gratuite à l'enseignement public, comme pour les Égyptiens, et leur fait payer des frais d'inscription

14 Depuis 2007, des centaines de personnes membres du mouvement Fatah sont en exil en Égypte, entre El Arich et Le Caire pour fuir la bande de Gaza. Suite à la guerre civile qui a opposé le Fatah et le Hamas, leur vie à Gaza était en danger. 
majorés, comme pour les étrangers. Ainsi Suheir, bien que née en Égypte, est considérée comme une étrangère dans son pays. Elle ne bénéficie donc pas de tous les droits dont jouissent les citoyens égyptiens à part entière. De plus, les conditions socio-économiques de ses parents ne lui permettaient pas l'accès à l'instruction publique égyptienne, encore moins à l'instruction privée : pour ses parents, la seule solution envisageable était alors que leur fille émigre à Gaza, où de la famille proche l'attendait et où elle pourrait suivre gratuitement un cursus scolaire. C'était donc la raison de leur présence au terminal de Rafah.

Cet événement s'explique en partie par le fait que, du point de vue légal, une partie des citoyens palestiniens qui vivent en Égypte possèdent un document octroyé par la République Arabe d'Égypte (et non pas par I'UNRWA ${ }^{15}$ ), le "Document de voyage pour les réfugiés palestiniens ": il s'agit d'une carte bleue qui se situe entre un passeport et une carte d'identité. L'utilité de ce document est très limitée : les Palestiniens en Égypte sont soumis d'une part à d'importantes restrictions de sortie du pays (la résidence leur est révoquée après six mois passés à l'étranger) et, d'autre part et en même temps, le renouvellement du "document de voyage " dépend du paiement d'une certaine somme et de la production d'un justificatif qui permet de prouver la présence dans le pays. Le fait ensuite que de nombreux Palestiniens ne puissent pas renouveler ce document génère la présence en Égypte d'un nombre croissant de Palestiniens "illégaux ", sans droits civils, non recensés : invisibles (El-Abed, 2009). Ce document est lui-même divisé en cinq sous catégories $(A, B, C, D, H)$ définies en fonction de la date d'arrivée dans le pays arabe, facteur qui détermine aussi la durée de validité du document et les modalités de renouvellement de celui-ci.

Comme nous pouvons le remarquer dans l'exemple que nous venons de présenter, la spatialisation de la frontière n'est pas un effet, mais une manifestation politique de la frontière même : plus il y a de déclinaisons et de fragmentations de la citoyenneté, plus la frontière rend spectaculaire sa propre action (De Genova et al., 2014 : 13) et se révèle décisive en termes de capacité d'organisation de l'espace et de la vie même des personnes.

\section{Conclusion}

La frontière que nous avons présentée est bien matérielle et bien visible : sa fonction explicite est d'imposer une forte discontinuité politique, géographique et de souveraineté entre les territoires. Mais bien plus en fragmentant le temps et l'espace, elle ne peut être interprétée selon une logique binaire comme le résultat de deux poussées opposées (Walker, $2006: 43$ et suiv.).

\footnotetext{
15 Selon I'UNRWA (Office de secours et de travaux des Nations Unies pour les réfugiés de Palestine dans le Proche-Orient), un réfugié de Palestine est " une personne dont le lieu de résidence habituelle était la Palestine entre juin 1946 et mai 1948, et qui a perdu à la fois sa maison et ses moyens de subsistance suite au conflit arabo-israélien de 1948. [...] La définition de réfugié couvre également les descendants des Palestiniens qui sont devenus des réfugiés en 1948 ». Actuellement I'UNRWA compte plus de 5 millions de réfugiés enregistrés, dont un tiers vit dans un des cinquante-huit camps de réfugiés (dont dix-neuf se trouvent en Cisjordanie et Jérusalem-Est, huit à Gaza, dix en Jordanie, neuf en Syrie et douze au Liban tandis qu'en Égypte où I'UNRWA n'est pas opérationnelle, il n'existe pas de camps de réfugiés palestiniens).
} 
Dès lors la question qui se pose n'est pas tant de savoir si et comment la frontière fonctionne, mais bien plutôt de comprendre ce que sont les effets de la frontière. II y a deux aspects à prendre en compte : I'espace social qui se crée " sur place " et la façon dont les effets de frontières se prolongent dans la vie des gens ; dimension horizontale de la production d'un espace social avec son autonomie partielle et dimension verticale de la temporalité des existences.

Los Tigres del Norte, un groupe californien de musique norteña ${ }^{16}$, chantaient dans le refrain d'une chanson populaire connue aux États-Unis : "Yo no crucé la frontera, la frontera me cruzó " ("Je n'ai pas traversé la frontière, la frontière $\mathrm{m}^{\prime}$ a traversé $\left."\right)^{17}$. En dépit du fait qu'à travers cet extrait Los Tigres del Norte revendiquaient plus leur "américanité ", en tant que véritables Amérindiens, que les gringos ou les WASP américains, nous pouvons retenir quelques éléments significatifs des paroles de leur chanson : dans certains cas, comme dans celui de la frontière mobile qui coupe en deux parties Rafah, la frontière traverse la vie des personnes sans que ces dernières soient disposées ou aptes à traverser la frontière. Dans de nombreux cas, tels que ceux décrits ici, la frontière traverse les personnes car elle laisse des traces sur leur corps, mais surtout dans leurs biographies. Ces personnes portent alors la frontière en elles, et coincées aux confins de l'intérieur et de l'extérieur, elles deviennent frontière (Khosravi, 2010 : 99).

La traversée ou non de la frontière n'en épuise pas la fonction, bien au contraire, et il semble que la persistance d'échanges transfrontaliers, et les traversées continues dans I'une ou l'autre direction, contribuent au maintien de la frontière, en amplifiant, en maintenant ou en créant de nouvelles hiérarchies, mais aussi des différences et des relations de pouvoirs (Parizot, 2006).

Au lieu de marquer une nette discontinuité entre intérieur et extérieur, entre États et souverainetés territoriales, la frontière fonctionne comme une membrane étendue dans le temps et dans l'espace, qui filtre, sélectionne, ordonne et gouverne les personnes, augmentant sa propre influence sur une région entière qui est amenée à se transformer en une zone-frontière.

Dans le cas de la frontière - linéaire, seulement en apparence - qui sépare la bande de Gaza de l'Égypte, nous pouvons saisir le " caractère radicalement ambigu des frontières et leur croissante incapacité de tracer une ligne stable entre l'intérieur et l'extérieur des territoires étatiques " (Mezzadra et Neilson, 2013 : 7).

16 La musique Norteña est un genre musical diffusé au nord du Mexique et aux ÉtatsUnis, surtout au niveau des communautés de Mexicains-Américains.

17 Los Tigres del Norte (2008) Somos más Americanos - (Uniendo fronteras), in Tu Noche con los Tigres del Norte, Fonovisa. 


\section{Références bibliographiques}

Andreas Peter (2004) Criminalized Legacies of War. The Clandestine Political Economy of the western Balkans, Problems of Post-Communism, 51 (3), pp. 3-9.

Anteby-Yemini Lisa (2008) Migrations africaines et nouveaux enjeux de la frontière israélo-égyptienne, Cultures \& Conflits, 72, pp. 77-99.

De Genova Nicholas, Mezzadra Sandro and Pickles John (Eds.) (2014) New Keywords: Migration and Borders, Cultural Studies, 29 (1), [en ligne]. URL : http://www.nicholasdegenova.net/resources/New+Keywords.pdf

El-Abed Oroub (2009) Unprotected: Palestinians in Egypt since 1948, Washington DC-Ottawa, International Development Research Centre-Institute for Palestine Studies, $253 \mathrm{p}$.

Hanafi Sari et Sammartin Olivier (1996) Histoires de frontières : les Palestiniens du Nord-Sinaï, Maghreb-Machrek, 151, pp. 51-66.

Khosravi Shahram (2010) "Illegal" traveller. An Auto-Ethnography of Borders, Basingstoke, Palgrave Macmillan, $150 \mathrm{p}$.

Mezzadra Sandro and Brett Neilson (2013) Border as method, or, the multiplication of labour, Durham-London, Duke University Press, 384 p.

Palidda Salvatore (2002) Come si studia il lavoro della polizia, in Alessando dal Lago e Rocco De Biasi Eds., Un certo sguardo. Introduzione all'etnografia sociale, Laterza, Bari, pp. 218-244.

Parizot Cédric (2009) Après le mur : les représentations israéliennes de la séparation avec les Palestiniens, Cultures \& Conflits, 73, pp. 53-72.

Parizot Cédric (2006) Crossing Borders, Retaining Boundaries. Kin-nections of Negev Bedouin in Gaza, the West Bank and Jordan, Cairo Papers in Social Science, 29 (1), pp. 58-84.

Rahola Federico (2003) Zone definitivamente temporanee. I luoghi dell'umanità in eccesso, Verona, Ombre corte, $236 \mathrm{p}$.

Vila Pablo (2000) Crossing borders, reinforcing borders. Social categories, metaphors, and narrative identities on the U.S.-Mexico frontier, Austin, University of Texas Press, 290 p.

Walker Rob B. J. (2006) L'enigma dell'internazionale, Conflitti Globali, 2, pp. 42-57. 